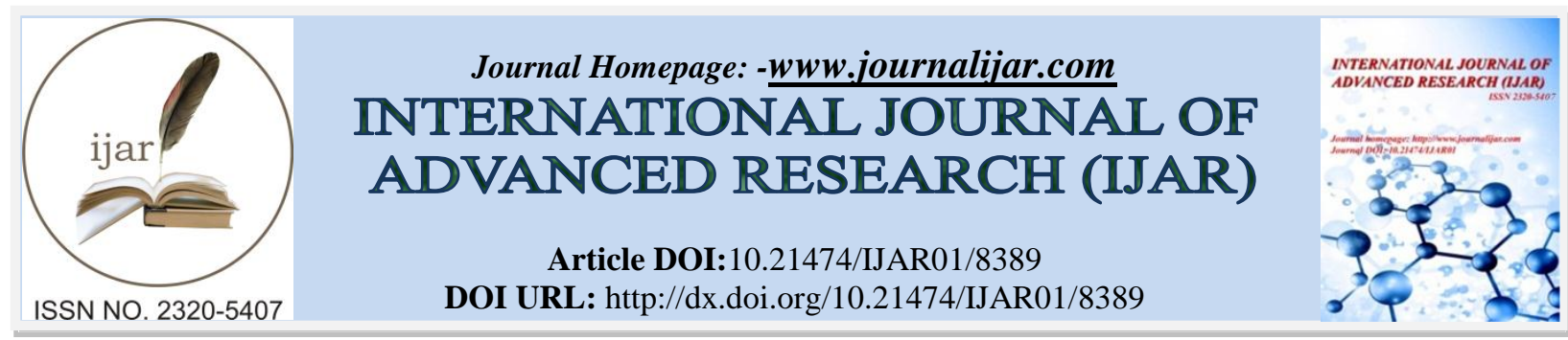

RESEARCH ARTICLE

\title{
SOLITARY INTRAMUSCULAR CYSTICERCOSIS MIMICKING MESENCHYMAL LESION: A RARE CASE REPORT.
}

Jayani Patel, Abhijit Das and MadhuSinha.

Department of Pathology, Janakpuri Super Speciality Hospital, New Delhi, India.

\section{Manuscript Info}

\section{Manuscript History}

Received: 12 November 2018

Final Accepted: 14 December 2018

Published: January 2019

\section{Abstract}

\section{Introduction:-}

1. Cysticercosis is a parasitic infection caused by encysted larvae of Taenia solium, the pork tapeworm. ${ }^{1}$ Tapeworm infections are common in developing countries like Africa, Asia, Latin America, where there is poor access to sanitation facilities and close interaction between humans and animals and where pigs are a major source of food. ${ }^{2,3}$ In India, it is more common in northern parts.

2. Moreover, cysticercosis is no longer an endemic disease of the developing countries, because of immigration from endemic areas, it has become a global problem. ${ }^{2,3}$

3. It is the most common parasitic infection of the nervous system in immunocompetent individuals along with muscle, eye and heart. ${ }^{4}$ The larvae form cysts commonly in the brain, meninges, and eyes, which together constitute $86 \%$ of the cases. Most soft tissue and muscular cysticercal involvement is associated with central nervous system involvement or multiple cysts. Solitary muscle cysticercosis without involvement of central nervous system is rare ${ }^{5,6}$ and difficult to diagnose due to lack of specific features. ${ }^{7}$

4. Here, we describe a case of intramuscular cysticercosis without any associated neurological or ocular involvement.

\section{Case Report}

A 12 years old male patient presented with a painless swelling over left arm. The swelling was gradually increasing in size. On examination, there was swelling measuring 4 × $3 \mathrm{cms}$ over anterior aspect of left arm. The swelling was firm, non- reducible with normal temperature. The overlying skin appeared normal.

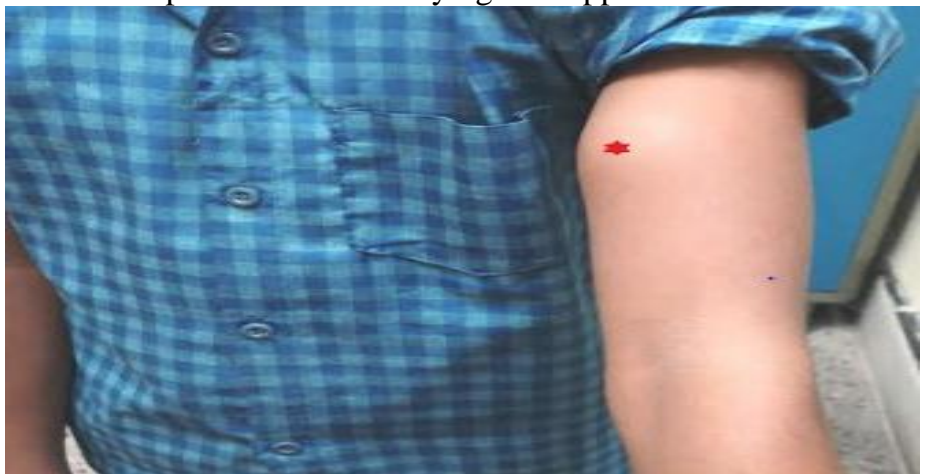

Image1:-A firm and non-reducible swelling over anterior aspect of left arm

Corresponding Author:-Jayani Patel.

Address:-Department of Pathology, Janakpuri Super Speciality Hospital, New Delhi, India. 
No other relevant history was present but residing in area where sanitation conditions were poor. Haematological parameters showed normal complete blood count. No other clinical abnormalities were found, with normal neurological and ophthalmological examinations.

FNA (Fine needle aspiration) from the swelling shows spindle to plump shaped cells along with multinucleated giant cells \& stromal fragments, inflammatory infiltrate comprising of lymphocytes and histiocytes suggestive of either infectious aetiology or mesenchymal lesion.

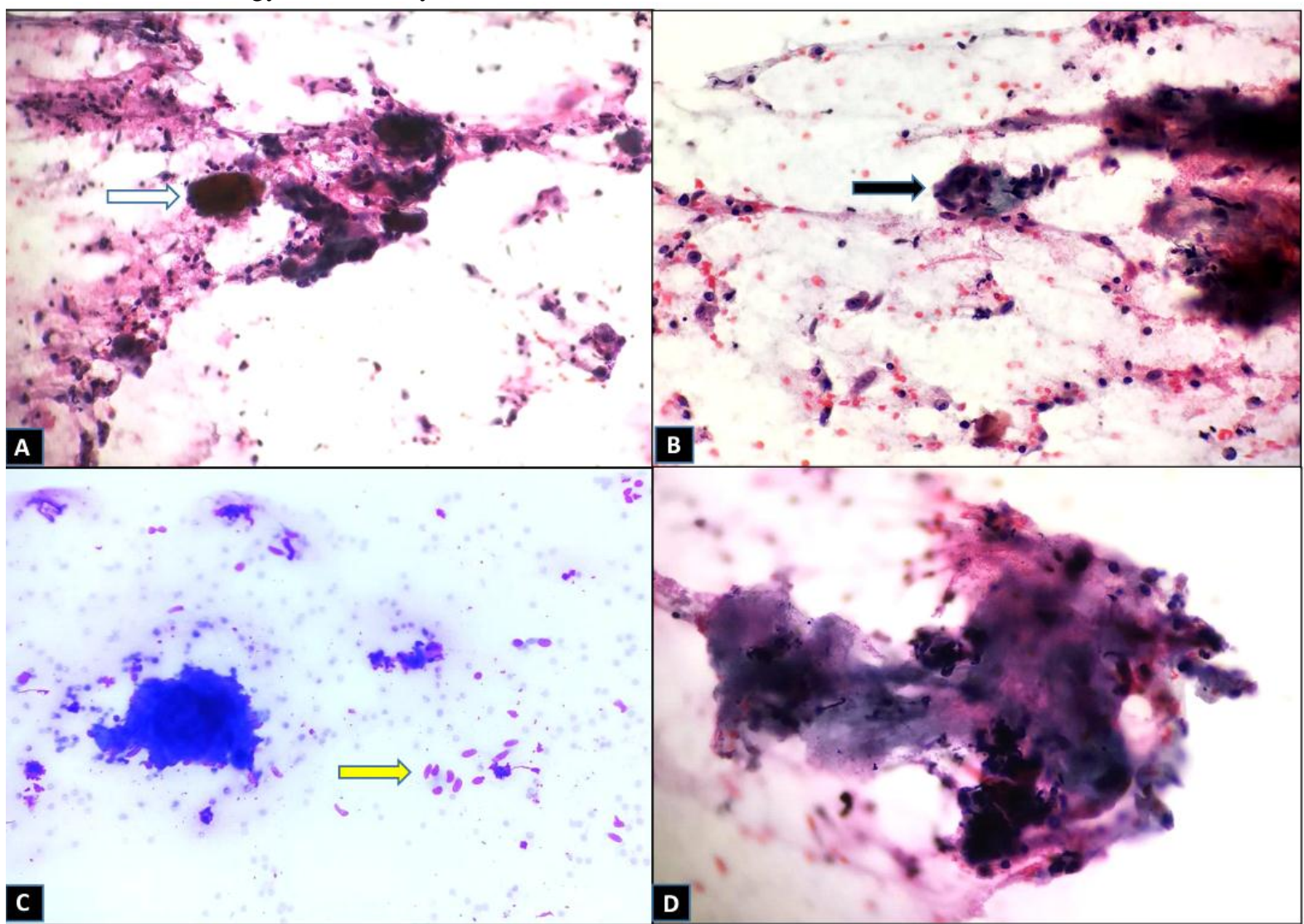

Figure 2 (A,B,C\&D):-Reactive muscle giant cells (white arrow), histiocytes\& inflammatory background (A); granuloma (black arrow) \& inflammatory background (B); benign spindle cells (yellow arrow) mimicking mesenchymal lesion (C); Fibrillary bluish material without any characteristic nuclei (? Fragment of bladder wall) (D).

The patient was sent to the Department of Radiology and Imaging for MRI.

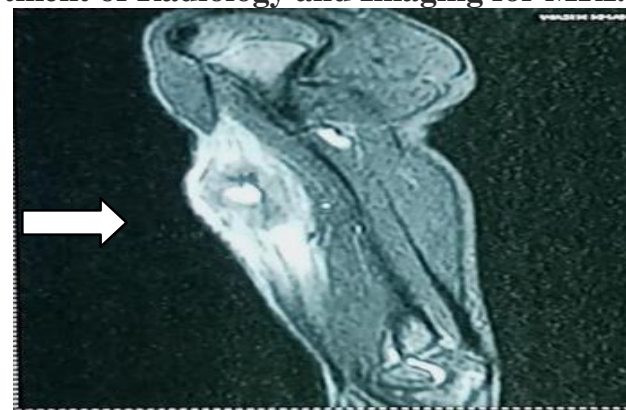

Image 3:-MRI findings: Focal rounded fluid intensity intramuscular lesion with peripheral hypointense rim located in biceps brachii muscle in the left upper arm

1. MRI findings are suggestive of cysticercus cyst/ evolving abscess in biceps brachii muscle.

2. The patient was managed conservatively with short course of tablet albendazole for 21 days. After three weeks of conservative treatment, on follow up, the swelling decreases in size. 


\section{Discussion:-}

Human cysticercosis is the infection caused by the larva of T. Solium, in which man is definite and pig is intermediate host.

When pigs or humans ingest the eggs, the gastric secretions break the outer wall of eggs. The oncospheres are released which attach and penetrate the intestinal wall. On the surface of oncospheres hooks and suckers are present which helps in attachment and penetration of intestinal wall. Oncospheres enter into the mesenteric venules and via bloodstream lodge in various tissues, and develop into cysts. ${ }^{8}$ Cysts may lodge anywhere in the body but brain, eyes, subcutaneous tissue and muscles, are more commonly affected. ${ }^{9}$ Other rare sites which have been reported are tongue, oral cavity, breast, heart and lungs. ${ }^{10,11,12}$

The encysted larva of T. solium called cysticercuscellulosae can remain viable in this stage for long time in humans; Living larvae evade immune recognition and do not elicit inflammation. This phase may last for years and is often clinically silent except when cyst location or size causes signs or symptoms. When the larva dies, it induces a vigorous acute inflammatory response that may produce symptoms, depending on the anatomic location. The resulting acute inflammation may result in local pain and myalgia. Alternatively, degeneration of the cyst may result in intermittent leakage of fluid, which elicits a chronic inflammatory response, with collection of fluid around the cyst, resulting in a mass-like, pseudotumor, or abscess-like lesion. Alternatively, the cyst retracts, its capsule thickens, and the scolexcalcifies. The cyst may be completely calcified later on. When the muscle burden of the cyst is large, pseudohypertrophy of the muscle results, characterized by multiple nodules. ${ }^{13-15}$ Such patterns are seen in hyperendemic areas.

People of any age may be affected but children commonly suffer because of increased chances of fomite infection. ${ }^{16}$ Solitary muscular and soft tissue cysticercal involvement is a rare disease and has been used as a marker of neurocysticercosis. Therefore, central nervous system or ocular involvement should be ruled out if systemic involvement is suspected. However, in our case no systemic involvement was there.

Diagnosis of solitary intramuscular cysticercosis is often delayed and missed due to nonspecific clinical presentations which mimicks various conditions like lipomas, epidermoid cysts, neuroma, neurofibromas, pseudoganglia, sarcoma, myxoma, pyomyositis, cold abscess or tuberculous lymphadenitis.

Various diagnostic modalities employed to detect cysticercosis include radiology, serology, and pathological examination. CT scan and MRI, though sensitive in diagnosing cysticercosis especially when the parasite involves the CNS, are very expensive. Moreover they provide only supportive diagnosis. Serological tests are useful if positive but cannot rule out the disease with negative results. False positivity is expected with the past parasitic infection or cross reactivity with other helminths.

The definitive diagnosis requires demonstration of the parasite. Fine needle aspiration cytology (FNAC) is rapid, safe, cheap, and reliable diagnostic tool for cysticercosis ${ }^{17}$ On cytology the inflammatory background ranges from acute inflammation from many eosinophils, neutrophils, palisading histocytes through epithelioid cell granulomas with giant cells to acellular necrosis without significant residual inflammation. ${ }^{18}$ Fragments of larval cuticle and parenchyma are strongly suggestive of cysticercosis. Hooklets are diagnostic which are present within the acute inflammatory exudate. However, the sample obtained may not be representative and many of the times the scolex and hooklets are missed.

So, FNAC smears showing mixed inflammatory cells infiltrate along with giant cells in varying proportions should be viewed with high index of suspicion, as it may be of parasitic origin, even in the absence of identifiable parasitic fragments and aspirated fluid. FNAC should be processed completely and should not be discarded as nonspecific. ${ }^{18}$ A diagnosis of cysticercosis is vital, as it calls for a search for the parasite in vital organs, where it causes significant morbidity and can even prove to be fatal. ${ }^{18}$

\section{Conclusion:-}

Although, solitary intramuscular cysticercosis is rare and generally asymptomatic in presentation, the possibility should be kept in mind in all inflammatory \&/or cystic intramuscular mass, especially in endemic areas like ours. FNAC in cysticercosis is a low-cost outpatient procedure. It is one of the tools for preoperative diagnosis and may even obviate the need for open biopsy. The cytological diagnosis is quite straightforward in cases where actual 
parasite structure is identified in the smears. However, in other cases, the presence of eosinophils, histiocytes which may be in palisaded clusters or not, a typical granular dirty background, etc., are the features which should always alert the pathologist to this possibility.

\section{References:-}

1. Del Brutto O.H., Sotelo J. Neurocysticercosis: an update. Rev Infect Dis. 1988;10(November-December (6)):10751087.

2. Kraft R. Cysticercosis: an emerging parasitic disease. Am Fam Physician. 2007;76(July (1)):91-96.

3. Garcia H.H., Del Brutto O.H. Taeniasolium cysticercosis. Infect Dis Clin N Am. 2000;14(March (1)):97-119.

4. Del Brutto O.H., Wadia N.H., Dumas M., Cruz M., Tsang V.C., Schantz P.M. Proposal of diagnostic criteria for human cysticercosis and neurocysticercosis. J Neurol Sci. 1996;142(October (1-2)):1-6.

5. Abdelwahab I.F., Klein M.J., Hermann G., Abdul-Quader M. Solitary cysticercosis of the biceps brachii in a vegetarian: a rare and unusual pseudotumor. SkeletRadiol. 2003;32(July (7)):424-428.

6. Zemeno-Alanis G.H. A classification of human cysticercosis. In: Fissler A., Willms K., Laclette J.P., editors. Cysticercosis: Present State of Knowledge and Perspectives. Academic Press; New York: 1982. pp. $107-127$.

7. Ogilvie C.M., Kasten P., Rovinsky D., Workman K.L., Johnston J.O. Cysticercosis of the triceps - an unusual pseudotumor: case report and review. ClinOrthopRelat Res. 2001;(January (382)):217-221.

8. Yamashita P., Kelsey J., Henderson S.O. Subcutaneous cysticercosis. J Emerg Med. 1998;16(July-August (4)):583586.

9. Mittal A., Das D., Iyer N., Nagaraj J., Gupta M. Masseter cysticercosis - a rare case diagnosed on ultrasound. DentomaxillofacRadiol. 2008;37(February (2)):113-116.

10. Kunkel J.M., Hawksley C.A. Cysticercosis presenting as a solitary dominant breast mass. Hum Pathol. 1987;18(November (11)):1190-1191.

11. Rao P.L., Radhakrishna K., Kapadia R.D. Cysticercosis of the tongue. Int J PediatrOtorhinolaryngol. 1990;20(November (2)):159-161.

12. Romero de Leon E., Aguirre A. Oral cysticercosis. Oral Surg Oral Med Oral Pathol Oral RadiolEndod. 1995;79(May (5)):572-577.

13. Asrani A, Morani A. Primary sonographic diagnosis of disseminated muscular cysticercosis. J Ultrasound Med. 2004;23(9):1245-1248.

14. Bandyopadhyay D, Sen S. Disseminated cysticercosis with huge muscle hypertrophy. Indian J Dermatol. 2009;54(1):49-51. doi: 10.4103/0019-5154.48987

15. Singal R, Mittal A, Gupta S, Gupta R, Sahu P, Gupta A. Intramuscular cysticercosis diagnosed on ultrasonography in thigh: A rare case report. N Am J Med Sci. 2010;2(3):162-164.

16. Ramraje S., Bhatia V., Goel A. Solitary intramuscular cysticercosis - a report of two cases. Australas Med J. 2011;4(1):58-60.

17. Adhikari RC, Aryal G, Jha A, Pant AD, Sayami G. Diagnosis of subcutaneous cysticercosis in fine needle aspirates: a study of 10 cases. Nepal Med Coll J. 2007;9:234-8.

18. Suchitha S, Vani K, Sunila R, Manjunath GV. "Fine Needle Aspiration Cytology of Cysticercosis-A Case Report," Case Reports in Infectious Diseases. 2012;2012:854-704. 